\title{
Upper Oesophageal Foreign Body with Acute Drooling in a Child with Congenital Hypotonia
}

\author{
AbdulWahab ${ }^{* 1,2}$, Kammouh $\mathrm{H}^{1}$ and Aljbawi WA ${ }^{3}$ \\ ${ }^{1}$ Department of Pediatrics Pulmonology, Hamad Medical Corporation, Qatar \\ ${ }^{2}$ Weill Cornell Medical College, Qatar \\ ${ }^{3}$ Department of Pediatrics Intensive Care, Hamad Medical Corporation, Qatar
}

*Corresponding author: AbdulWahab A, MD, FCCP, Senior Consultant Pediatric Pulmonologist, Hamad Medical Corporation, Assistant Professor of Clinical Pediatrics, Weill Cornell Medicine College, Qatar, Tel: 0097466005065, E-mail: aabdulwahab2@hamad.qa, atqah2015@gmail.com

Citation: AbdulWahab A, Kammouh H, Aljbawi WA (2017) Upper Oesophageal Foreign Body with Acute Drooling in a Child with Congenital Hypotonia. J Case Rep Stud 5(5): 505

Received Date: September 16, 2017 Accepted Date: October 26, 2017 Published Date: October 28, 2017

\begin{abstract}
Foreign body ingestion and aspiration commonly affect children. The most vulnerable age is early childhood when children tend to explore new objects with their mouths or by an accident by elder siblings. A 2-year-old female child who is known to have congenital hypotonia, status post laparoscopic fundoplication and gastrostomy tube feeding (G- tube) inserted at age one year. Then she was in stable condition till when presented with acute unexplained drooling of frothy whitish secretions, mild cough and difficulty breathing with hypoxemia. X-ray chest and upper abdomen revealed normal. She underwent flexible bronchoscopy revealed normal and then direct laryngoscopy was extracted a covering plastic of the feeding tube in the upper end cervical oesophagus by Magill forceps technique. Subsequently her symptoms resolved completely. To increase the likelihood of identifying foreign body ingestion and aspiration, healthcare professionals should maintain a high index of suspicion even in children with neuromuscular disorder.
\end{abstract}

Keywords: Foreign Body; Congenital Hypotonia; Drooling; Magill Forceps

\section{Introduction}

Foreign body ingestion and aspiration commonly affect children [1]. The most vulnerable age is between six months and four years [2]. We report her unexplained acute drooling in a child with congenital hypotonia and healthcare professionals should maintain a high index of suspicion of foreign body ingestion.

\section{Case Report}

Our case is a 2-year-old female child who is known to have congenital hypotonia, gastroesophageal reflux disease post laparoscopic fundoplication and gastrostomy tube feeding (G- tube) started at age one year. She was doing very well with no episodes of choking or drooling or recurrent aspiration in last one year. She presented to paediatrics casualty with a 4-hour history of acute drooling of frothy whitish secretions, mild cough and difficulty breathing with oxygen saturation 85-88\% in room air. No history of fever or $\mathrm{flu}$-like illness. Her mother is a medical nurse and noted there were missing small toys from her elder sister, and she insisted her presentation was not usual and most probably either ingested or aspirated foreign body. She was stabilized with frequent suction and oxygen therapy via flow nasal cannula and transferred to the paediatric intensive care unit. Radiographic Study: Both chest $\mathrm{X}$-ray and upper abdomen revealed normal and no radiopaque foreign body.

\section{Airway Evaluation}

As the patient was tachypneic, coughing with some drooling and breathing oxygen at 2 liters per minute by nasal prongs. So initially the fiberoptic bronchoscope was performed and introduced transnasally into the posterior pharynx and vocal cords were visualized. The cords moved well. The bronchoscope was then passed through the cords and the tracheobronchial tree was inspected. The trachea, segmental areas of the right upper lobe, right middle lobe, right lower lobe, left upper lobe, lingula and left lower lobe were patent with no airway compression or abnormalities or foreign bodies. Immediately after the procedure, the patient continued to have drooling and possibility of foreign body ingestion in hypopharynx and upper esophageal. She underwent 
direct laryngoscopy and extracted covering plastic of the feeding tube (Figure 1) in the upper end cervical esophagus by Magill forceps technique. The child was discharged after few hours in satisfactory general condition.

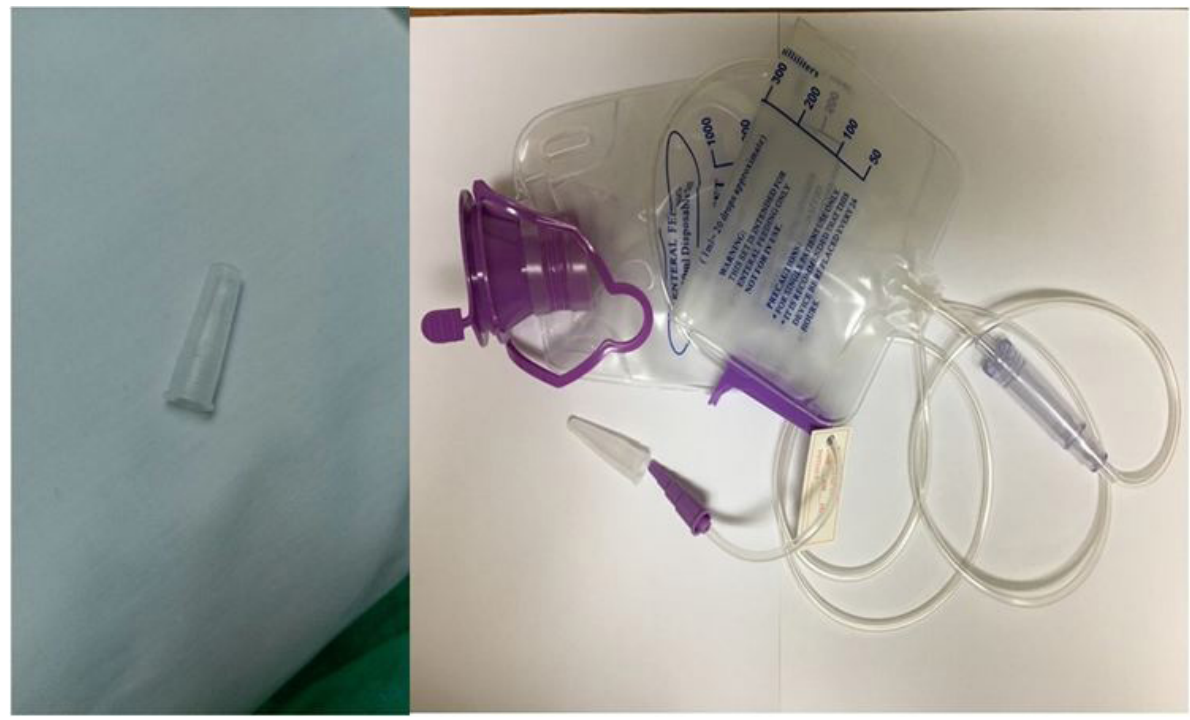

Figure 1: Feeding tube with covering plastic

\section{Discussion}

Foreign body ingestion is a common emergency problem in children and may cause morbidity and mortality in the paediatric population [3]. The most frequently ingested foreign bodies in children are coins, toys, magnets, batteries and other ingested objects include jewels, needles, pins, balls and buttons [4]. Most of the FBs were located in the upper oesophagus (68\%). Success rate for retrieval was greater for oesophageal FBs (99\%) than for more distally located FBs (70\%; P < 0.001) [5]. To our knowledge, this is the first reported case of a covering plastic of the enteral tube feeding ingestion that get stuck at the level of upper end cervical oesophagus in a child with congenital myopathy. This may be related to the dynamics of the swallowing in hypotonic child; possibility due to uncoordinated swallowing mechanisms in young children and impairment of the cricopharyngeal muscle function and its ability to relax, which then prevents the food from leaving the hypopharynx into the esophagus. Such Sharp objects have a tendency to get stuck at the level of upper oesophagus causing both respiratory and GIT symptoms.

The diagnosis of oesophageal foreign body ingestion may be as simple as taking a history or may be challenging by acute unusual symptoms, a high level of suspicion is needed especially in cases where ingestion of a foreign body like life-threatening airway compromise or unexplained excessive drooling with difficulty breathing as in our case with neuromuscular disability [6]. It has been reported in the literature an 8-year-old boy who presented with a cough, mild respiratory difficulty and drooling. A marble ball was found just above the thoracic inlet, pushing the posterior wall of the trachea anteriorly causing narrowing of the lumen [5].

In our case the non-radiopaque plastic cover was successfully removed by direct laryngoscopy and Magill's forceps under propofol sedation without any complications. Several studies demonstrated direct vision or laryngoscopy-aided view could be enough to make the diagnosis and extract the foreign body in the upper oesophagus by using Magill forceps. Such technique minimizes instrumentation of the oesophagus and is an easy, safe technique for removing coins that stuck in the upper end of the oesophagus [7-9].

Prevention of foreign body ingestion in children is a high priority. Caregivers should be educated about preventing small children from contact with small objects that may be harmful if swallowed, especially any plastic cover as in our case, button/disc batteries, small magnets, and other high-risk objects. This includes items placed into a trash container that may make accessible to children. The possibility of acute unusual or non-specific symptoms being caused by foreign body ingestion should always be kept in mind.

\section{Conclusion}

A high index of suspicion must be maintained and early recognition of foreign body ingestions in children with neuromotor disabilities. An appropriate management can significantly reduce morbidity due to complications.

\section{References}

1. Louie MC, Bradin S (2009) Foreign body ingestion and aspiration. Pediatr Rev 30: 295-301.

2. Klein CA (2012) Intentional ingestion and insertion of foreign objects: a forensic perspective. J Am Acad Psychiatry Law 40: 119-26.

3. Diaconescu S, Gimiga N, Sarbu I, Stefanescu G, Olaru C, et al. (2016) Foreign Bodies Ingestion in Children: Experience of 61 Cases in a Pediatric Gastroenterology Unit from Romania. Gastro Res Pract 2016: 1982567 doi: 10.1155/2016/1982567.

4. Sugawa C, Ono H, Taleb M, Lucas CE (2014) Endoscopic management of foreign bodies in the upper gastrointestinal tract: A review. World J Gastrointest Endosc 6: 475-81. 
5. Urkin J, Bar-David Y (2006) Respiratory distress secondary to esophageal foreign body: a case report. ScientificWorldJournal 6: 16-9.

6. Lao VV, Lusting D, Boseley M, Lao OB (2015) Pediatric ingested foreign body, acquired tracheoesophageal fistula-endoscopic repaire with cautery \& fibrin glue (Tisseel): case report and literature review. J Pediatr Surg Cas Rep 3: 426-31.

7. Baral BK, Joshi RR, Bhattarai BK, Sewal RB (2010) Removal of coin from upper esophageal tract in children with Magill's forceps under propofol sedation. Nepal Med Coll J 12: 38-41.

8. Cetinkursun S, Sayan A, Demirbag S, Surer I, Ozdemir T, et al. (2006) Safe removal of upper esophageal coins by using Magill forceps: two centers' experience. Clin Pediatr (Phila) 45: 71-3.

9. Arana A, Hauser B, Hachimi-Idrissi S, Vandenplas Y (2001) Management of ingested foreign bodies in childhood and review of the literature. Eur J Pediatr 160: 468-72.

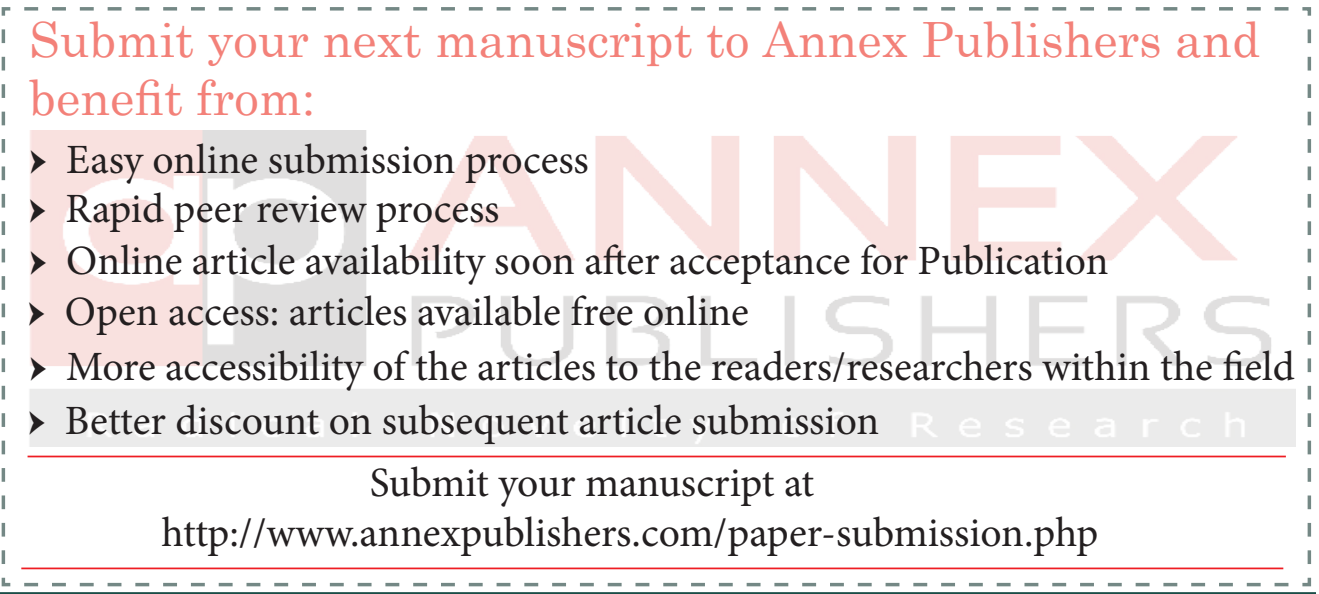

